Supporting Information

\title{
Mechanistic Model for the Prediction of Small-Molecule Vitreal Clearance Combining Diffusion-Limited and Permeability-Limited Clearance
}

Anna-Kaisa Rimpelä*, Yunhai Cui, Achim Sauer

Department of Drug Discovery Sciences, Boehringer Ingelheim Pharma GmbH \& Co. KG, 88397 Biberach, Germany

*Correspondence: anna-kaisa.rimpelae@boehringer-ingelheim.com

Table S1. Permeability of eight model compounds included in our data set measured in RPE models. ${ }^{1,2}$

\begin{tabular}{lccccccccc}
\hline & \multicolumn{8}{c}{ Permeability $\left.\times \mathbf{8} \mathbf{1 0}^{-6} \mathbf{c m} / \mathbf{s}\right)$} \\
\hline & BB & Bovine RPE-choroid & \multicolumn{2}{c}{ ARPE-19 $^{2}$} & \multicolumn{3}{c}{ LEPI $^{2}$} & \multicolumn{2}{c}{ hESC-RPE $^{2}$} \\
\hline Aztreonam & 5.4 & 4.5 & 6.5 & 11 & 1.9 & 0.40 & & \\
Ciprofloxacin & 9.5 & 1.4 & 10.2 & 17 & 2.3 & 0.59 & 5.6 & 3.0 \\
Fluconazole & 16 & 13 & & & 8.0 & 5.4 & 23 & 20 \\
Ganciclovir & 9.7 & 6.5 & & & 2.3 & 0.87 & 2.8 & 0.99 \\
Ketorolac & 69 & 4.8 & 15 & 18 & 3.8 & 1.3 & 12 & 6.9 \\
Methotrexate & 9.4 & 4.5 & 8.4 & 13 & 1.3 & 0.44 & 1.6 & 0.37 \\
Quinidine & & & & & & & 13 & 14 \\
Voriconazole & 25 & 21 & & & & & 32 & 28 \\
\hline
\end{tabular}

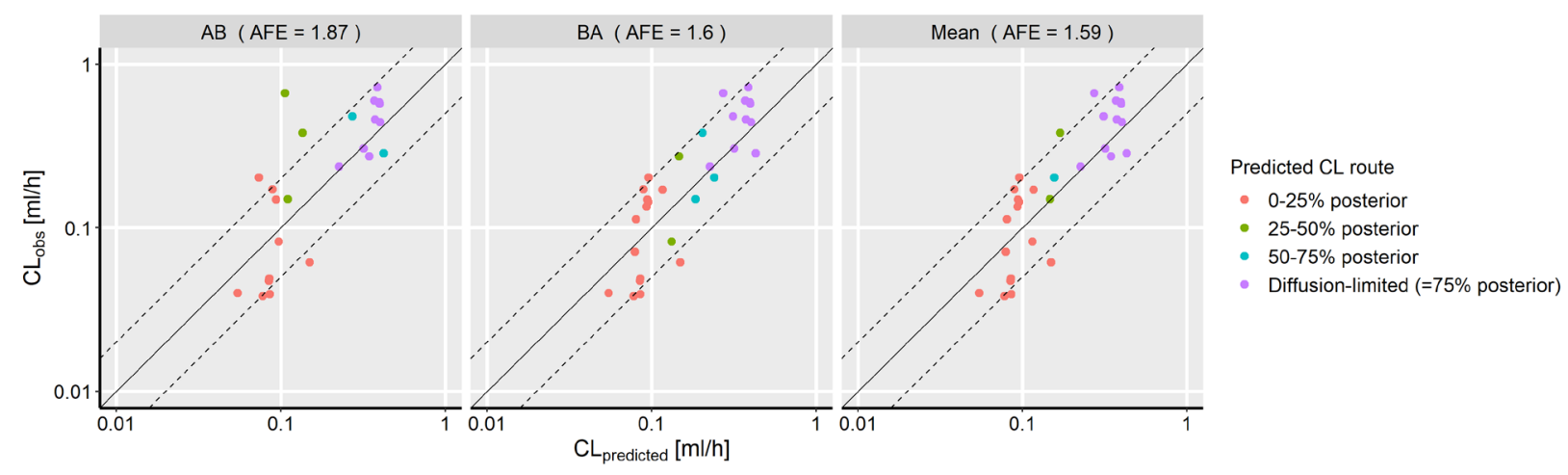

Figure S1. Comparison of predicted vitreal clearance $(C L)$ using the apical-to-basolateral $(A B)$, basolateralto-apical (BA) and intrinsic (Mean) Caco-2 permeabilities with volume-corrected observed $\mathrm{CL}$. In the $A B$ direction, the compounds with only an upper limit for permeability available, were excluded. 
Table S2. Compound info, permeabilities, observed and predicted $\mathrm{CL}$ and vitreal volume of distribution ( $\left.\mathrm{V}_{\text {vit }}\right)$ reported in literature.

\begin{tabular}{|c|c|c|c|c|c|c|c|c|c|c|c|c|}
\hline Compound & MW & $\mathbf{P}_{\mathrm{app}, \mathrm{int}}$ & $\begin{array}{c}\mathbf{P}_{\mathrm{app}, \mathbf{A B}} \\
\left(\times 10^{-6} \mathrm{~cm} / \mathrm{s}\right)\end{array}$ & $\mathbf{P}_{\mathrm{app}, \mathrm{BA}}$ & ER & $\begin{array}{l}\mathrm{CL}_{\text {obs }} \\
(\mathrm{ml} / \mathrm{h})\end{array}$ & $\begin{array}{c}\text { Original } \mathbf{V}_{\text {vit }} \\
(\mathrm{ml})\end{array}$ & $\mathrm{CL}_{\text {obs, corr }}$ & $\mathrm{CL}_{\text {pred, int }}$ & $\begin{array}{c}\mathrm{CL}_{\text {pred, }} \mathrm{AB} \\
(\mathrm{ml} / \mathrm{h})\end{array}$ & $\mathrm{CL}_{\text {pred, BA }}$ & $C L_{\text {perm,int }}$ \\
\hline Amikacin & 586 & $* *$ & & & & 0.035 & 1.37 & 0.038 & 0.078 & 0.078 & 0.078 & 0.0016 \\
\hline Aztreonam & 435 & $*$ & $<2.2$ & $<0.44$ & & 0.125 & 1.39 & 0.135 & 0.093 & & 0.093 & 0.0069 \\
\hline Cefazolin & 455 & $*$ & $<4.1$ & $<0.68$ & & 0.165 & 1.72 & 0.144 & 0.095 & & 0.095 & 0.011 \\
\hline Ceftazidime & 547 & $*$ & $<0.24$ & $<0.045$ & & 0.07 & 1.47 & 0.071 & 0.079 & & 0.079 & 0.0007 \\
\hline Ceftriaxone & 555 & $*$ & $<0.76$ & $<0.16$ & & 0.109 & 1.45 & 0.113 & 0.080 & & 0.080 & 0.0024 \\
\hline Cefuroxime & 424 & 18 & 32 & 3.8 & 0.12 & 0.235 & 1.29 & 0.273 & 0.344 & 0.344 & 0.146 & 0.280 \\
\hline Ciprofloxacin & 331 & 4.7 & 2.5 & 6.8 & 2.7 & 0.336 & 1.32 & 0.382 & 0.170 & 0.136 & 0.203 & 0.073 \\
\hline Clarithromycin & 748 & 33 & 2.4 & 63 & 27 & 0.32 & 0.72 & 0.667 & 0.272 & 0.106 & 0.272 & 0.510 \\
\hline Cyclosporine A & 1203 & 15 & 14 & 17 & 1.2 & 0.484 & 3.06 & 0.237 & 0.225 & 0.225 & 0.225 & 0.239 \\
\hline Diclofenac & 296 & 96 & 82 & 110 & 1.3 & 0.932 & 3.16 & 0.442 & 0.401 & 0.401 & 0.401 & 1.504 \\
\hline Erythromycin & 734 & 5.5 & 0.24 & 11 & 45 & 0.19 & 1.4 & 0.204 & 0.156 & 0.073 & 0.239 & 0.087 \\
\hline Fluconazole & 306 & 36 & 37 & 36 & 1.0 & 0.753 & 1.93 & 0.585 & 0.396 & 0.396 & 0.396 & 0.570 \\
\hline Foscarnet & 126 & 0.034 & 0.035 & 0.032 & 0.91 & 0.057 & 1.39 & 0.062 & 0.149 & 0.149 & 0.149 & 0.0005 \\
\hline Ganciclovir & 255 & $*$ & $<2.3$ & $<0.54$ & & 0.153 & 1.34 & 0.171 & 0.117 & & 0.117 & 0.0084 \\
\hline Gentamicin & 464 & $* *$ & & & & 0.031 & 1.18 & 0.039 & 0.085 & 0.085 & 0.085 & 0.0016 \\
\hline Hesperetin & 302 & 56 & 50 & 61 & 1.2 & 0.501 & 1.31 & 0.574 & 0.398 & 0.398 & 0.398 & 0.868 \\
\hline Indomethacin & 358 & 93 & 87 & 98 & 1.1 & 0.6 & 1.5 & 0.600 & 0.370 & 0.370 & 0.370 & 1.447 \\
\hline Ketorolac & 255 & 21 & 20 & 22 & 1.1 & 0.283 & 1.48 & 0.287 & 0.428 & 0.421 & 0.428 & 0.328 \\
\hline Lincomycin & 407 & 1.7 & $<0.55$ & 2.8 & & 0.0825 & 1.5 & 0.083 & 0.115 & 0.097 & 0.132 & 0.026 \\
\hline Methicillin & 380 & 0.15 & 0.13 & 0.17 & 1.3 & 0.218 & 2.19 & 0.149 & 0.093 & 0.093 & 0.094 & 0.0023 \\
\hline Methotrexate & 454 & 0.29 & 0.28 & 0.30 & 1.1 & 0.197 & 1.72 & 0.172 & 0.089 & 0.089 & 0.089 & 0.0045 \\
\hline Netilmicin & 476 & $* *$ & & & & 0.055 & 1.74 & 0.047 & 0.084 & 0.084 & 0.084 & 0.0016 \\
\hline Novobiocin & 613 & 4.6 & 2.2 & 7.0 & 3.1 & 0.15 & 1.5 & 0.150 & 0.147 & 0.110 & 0.184 & 0.072 \\
\hline Oxacillin & 401 & $*$ & $<1.9$ & 0.43 & & 0.31 & 2.29 & 0.203 & 0.096 & & 0.096 & 0.0066 \\
\hline Quinidine & 324 & 51 & 43 & 59 & 1.4 & 0.448 & 0.93 & 0.723 & 0.386 & 0.386 & 0.386 & 0.796 \\
\hline Tobramycin & 468 & $* *$ & & & & 0.04 & 1.22 & 0.049 & 0.085 & 0.085 & 0.085 & 0.0016 \\
\hline Vancomycin & 1449 & $* *$ & & & & 0.039 & 1.46 & 0.040 & 0.055 & 0.055 & 0.055 & 0.0016 \\
\hline Voriconazole & 349 & 90 & 110 & 70 & 0.64 & 0.421 & 1.37 & 0.461 & 0.374 & 0.374 & 0.374 & 1.408 \\
\hline BI1 & 540 & 30 & 12 & 48 & 3.9 & 0.48 & 1.5 & 0.480 & 0.311 & 0.271 & 0.311 & 0.472 \\
\hline $\mathrm{BI} 2$ & 515 & 78 & 65 & 91 & 1.4 & 0.305 & 1.5 & 0.305 & 0.317 & 0.317 & 0.317 & 1.219 \\
\hline
\end{tabular}

*Upper limit of the BA permeability used for the prediction.

**Known low permeability compounds, permeability set to $0.1 \times 10^{-6} \mathrm{~cm} / \mathrm{s}$ for the prediction, a lower permeability would have minimal impact on the prediction (<3\%). 

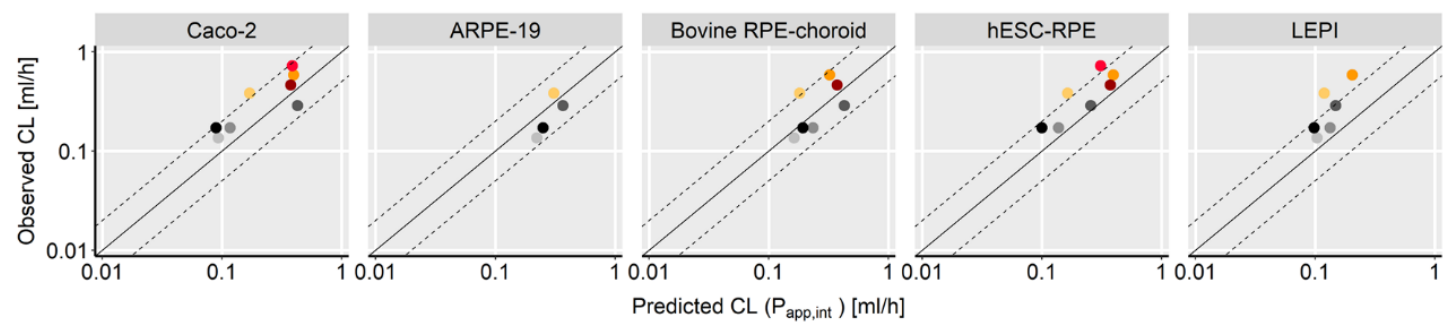

Compound

- Aztreonam

- Ganciclovir

- Ketorolac
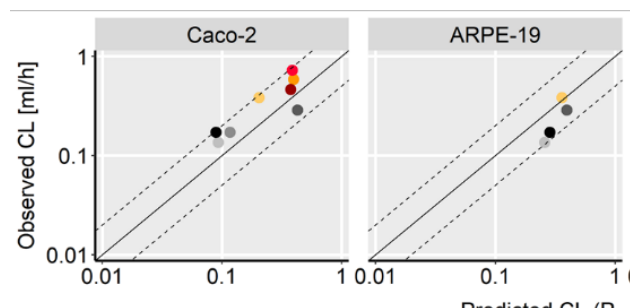
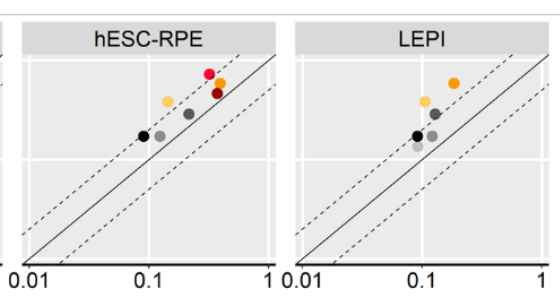

Ciprofloxacin

- Fluconazole

- Quinidine

- Voriconazole

Figure S2. Prediction of vitreal $\mathrm{CL}$ of model compounds using intrinsic and $\mathrm{AB}$ or BA permeability data from different in vitro models. The solid line is the unity line and the dashed lines represent 2-fold deviation. The first four compounds (grey-shaded colors) have a low maximum possible fold error $(<3.0)$.

\section{Model predictivity of literature QSPR models for vitreal CL or half-life}

To estimate the predictivity of literature vitreal pharmacokinetic (PK) prediction models, we predicted the corresponding PK parameter used in each publication for the compound data set of del Amo et al. ${ }^{3}$ including compounds with molecular weight in the range 100-1500 Da. Additionally, we included the five additional compounds in our study (indomethacin, lincomycin, novobiocin, BI1, BI2) in the estimation. For the assessment of the model reported by Kidron et al. ${ }^{4}$ and Durairaj et al. ${ }^{5}$, we also included the compounds reported in Kidron et al., as they were both predicting half-life. Many of the compounds reported by Durairaj et al. were part of the sets of del Amo and Kidron, therefore, no compounds were added separately from their data set.

Del Amo et al. ${ }^{3}$ reported two models describing vitreal $\mathrm{CL}$. Model a was based on hydrogen bond donors (HD), lipophilicity $\left(\log D_{7.4}\right)$ and polar surface area (PSA) $\left(\right.$ LogCL $_{\text {ivt }}=-0.17411-0.38180($ LogHD $)-0.00117$ PSA + 0.03686 $\left.\left(\log D_{7.4}\right)\right)$. Model b was based on HD and $\log \mathrm{D}_{7.4}\left(\operatorname{LogCL}_{\text {ivt }}=-0.25269-0.53747(\operatorname{LogHD})+\right.$ $\left.0.05189\left(\log _{7.4}\right)\right)$. Durairaj et al. ${ }^{5}$ reported multiple models but we chose the model which excluded intravitreal suspension data $\left(\log t_{1 / 2}=-0.240+0.281 \log (M W)-0.107 \log D_{7}\right)$. From Kidron et al. ${ }^{4}$, we used the model constructed with data from both albino and pigmented rabbits using lipophilicity $\left(\log \mathrm{D}_{7.4}\right)$ and total number of putative hydrogen bonds $\left(H_{\text {tot }}\right)\left(\log t_{1 / 2, \text { mixed }}=-0.046-0.051 \log D_{7.4}+0.640 \log \left(H_{\text {tot }}\right)\right)$. 

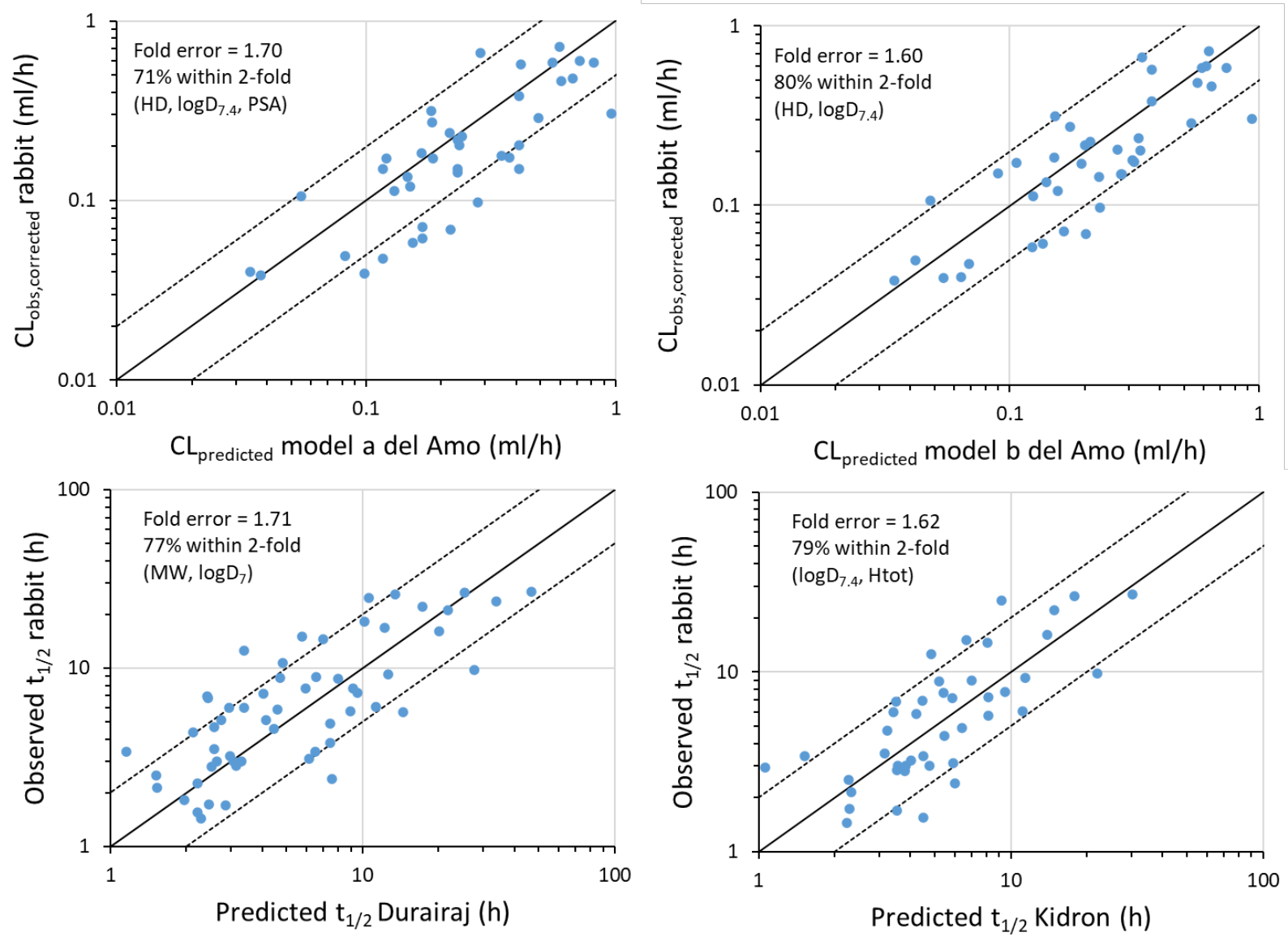

Figure S2. Model predictivity of QSPR models from del Amo et al. ${ }^{3}$, Durairaj et al. ${ }^{5}$ and Kidron et al. ${ }^{4}$. The solid line is the unity line and the dashed lines represent 2-fold deviation.

\section{References}

(1) Ramsay, E.; Hagström, M.; Vellonen, K.-S.; Boman, S.; Toropainen, E.; Amo, E. M. del; Kidron, H.; Urtti, A.; Ruponen, M. Role of Retinal Pigment Epithelium Permeability in Drug Transfer between Posterior Eye Segment and Systemic Blood Circulation. Eur J Pharm Biopharm 2019, 143, 18-23.

(2) Hellinen, L.; Hongisto, H.; Ramsay, E.; Kaarniranta, K.; Vellonen, K.-S.; Skottman, H.; Ruponen, M. Drug Flux Across RPE Cell Models: The Hunt for An Appropriate Outer Blood-Retinal Barrier Model for Use in Early Drug Discovery. Pharm 2020, 12 (2), 176.

(3) del Amo, E. M.; Vellonen, K.-S.; Kidron, H.; Urtti, A. Intravitreal Clearance and Volume of Distribution of Compounds in Rabbits: In Silico Prediction and Pharmacokinetic Simulations for Drug Development. Eur J Pharm Biopharm 2015, 95, 215-226.

(4) Kidron, H.; del Amo, E. M.; Vellonen, K. S.; Urtti, A. Prediction of the Vitreal Half-Life of Small Molecular Drug-like Compounds. Pharmaceut Res 2012, 29 (12), 3302-3311. 
(5) Durairaj, C.; Shah, J. C.; Senapati, S.; Kompella, U. B. Prediction of Vitreal Half-Life Based on Drug Physicochemical Properties: Quantitative Structure-Pharmacokinetic Relationships (QSPKR).

Pharmaceut Res 2009, 26 (5), 1236. 